\title{
Room Temperature Synthesis of Aminocaproic Acid-Capped Lead Sulphide Nanoparticles
}

\author{
Jayesh D. Patel $^{1,2}$, Frej Mighri ${ }^{1,2 *}$, Abdellah Ajji ${ }^{1,3}$, Saïd Elkoun ${ }^{1,4}$ \\ ${ }^{1}$ Center for Applied Research on Polymers and Composites (CREPEC), Montreal, Canada; ${ }^{2}$ Chemical Engineering Department, Laval \\ University, Quebec, Canada; ${ }^{3}$ Chemical Engineering Department, École Polytechnique de Montréal, Succursale Centre-Ville, Mont- \\ real, Canada; ${ }^{4}$ Mechanical Engineering Department, Sherbrooke University, Sherbrooke, Canada. \\ Email: *Frej.Mighri@gch.ulaval.ca
}

Received December $8^{\text {th }}, 2011$; revised January $17^{\text {th }}, 2012$; accepted January $28^{\text {th }}, 2012$

\begin{abstract}
Aminocaproic acid (ACA) mixed methanolic lead acetate-thiourea (PbAc-TU) complex as precursor for fabrication of lead sulphide ( $\mathrm{PbS}$ ) nanoparticles (NPs) has been explained. The size, structure and morphology of as-prepared ACAcapped PbS NPs were systematically characterized by scanning electron microscopy (SEM), Transmission electron microscopy (TEM), X-ray diffraction (XRD), Uv-vis spectroscopy and Brunauer-Emmett-Teller (BET) techniques. The obtained results show that the synthesized PbS NPs are nanocrystalline, size quantized and their agglomeration shows a mesoporous network of $8.7 \mathrm{~nm}$ in pore size. The binding nature of ACA molecules on PbS surface was studied by thermo gravimetric analysis (TGA), Fourier transform infrared (FTIR) and X-ray photoelectron (XPS) techniques. Results indicate that ACA acts as a soft template that restricts the growth of PbS NPs through its binding to Pb surface via nitrogen lone pair.
\end{abstract}

Keywords: Semiconductor; Nanoparticles; Lead Sulphide; Mesoporous; BET; XPS; FTIR

\section{Introduction}

In recent years, semiconducting nanoparticles have been studied frequently because of their highly tunable properties obtained by controlling their size, shape and surface morphology [1-4]. Being an important IV-VI group of semiconductors, $\mathrm{PbS}$ has attracted an increasing interest and has been extensively investigated due to its unique size quantization properties. Bulk PbS having cubic crystal structure and narrow direct energy band gap $(0.41 \mathrm{eV}$ at $300 \mathrm{~K}$ ) has a large excitonic Bohr radius (around 18 $\mathrm{nm})$. Hence, PbS NPs exhibit strong quantum size effects for relatively large sizes. Also, their absorption edge can be tuned to anywhere between near infrared to violet $(0.4$ $\mu \mathrm{m})$ covering the entire visible spectrum [5]. The combination of such properties makes PbS suitable for efficient electroluminescent devices, such as inorganic-organic bulk hybrid solar cells [6,7], tunable near-infrared detectors [8], and solid state lasers [9]. Further, at a given particle size, the third-order nonlinear optical response of PbS NPs is 30 times greater than that of gallium arsenide (GaAs) and 1000 times greater than that of cadmium sulphide (CdSe), which enables them to be a potential candidate for photonic and optical switching applications [10]. So far, a variety of PbS architectures have been sy-

${ }^{*}$ Corresponding author. nthesized by various methods. These structures include nanotubes, nanowires, cubes, octahedrons, flower structures, sheet-like shape, dendrite and macrostar-like hierarchical structures. Synthesis techniques generally include hydrothermal, solvothermal, chemical or thermal decomposition routes with conventional surfactants or capping molecules that act as a directing factor to regulate the size and the shape of the synthesized NPs [11-16].

Recently, biomolecule-assisted synthesis has been highly promising due to its novelty and its environmentally friendly character for a large variety of nanomaterials and also for its strong utility in morphology control [17]. To the best of our knowledge, only few references on the synthesis of $\mathrm{PbS}$ nanostructures using biomolecules are available in literature [17-20]. Aminocaproic acid (ACA, Scheme 1), a derivative of the amino acid lysine, is very effective in the treatment of bleeding disorders. It has functional groups, such as $-\mathrm{NH}_{2}$ and $-\mathrm{COOH}$ (Scheme 1), which have a strong capability of coordination with metal ions surface. It was successfully used to produce size and shape controlled NPs including their organized<smiles>NCCCCCCC(=O)O</smiles>

Scheme 1. Structure of aminocaproic acid (ACA). 
self-assembly nanostructures [21].

In the present contribution, $\mathrm{PbS}$ NPs were synthesized via an easily and rapid technique using ACA mixed $\mathrm{Pb}$ Ac-TU complex as a precursor. Utilization of this precursor allows a simple route for the synthesis of well-dispersed PbS NPs at room temperature compared to previously reported synthetic route for the synthesis of $\mathrm{PbS}$ NPs using PbAc-TU complex by microwave and sonochemical techniques [22]. Physical, optical and surface properties of the as-prepared PbS NPs show that these NPs are nanocrystalline and size quantized. ACA molecules work as a soft template and are bound to $\mathrm{Pb}$ surface through nitrogen lone pair.

\section{Experimental}

\subsection{Reagents}

All chemical used were of analytical grade and used without further purification. ACA, lead acetate trihydrate and thiourea were purchased from Sigma-Aldrich, Canada. Methanol was purchased from Fisher chemicals, Canada.

\subsection{Synthesis ACA-Capped PbS NPs}

In a typical synthesis, methanolic solution of PbAc-TU complex was prepared by dissolving 0.01 mole (M) $(0.189 \mathrm{~g})$ of lead acetate trihydrate and $0.01 \mathrm{M}(0.038 \mathrm{~g})$ of thiourea one by one in $50 \mathrm{ml}$ of methanol. The solution was stirred for $5 \mathrm{~min}$ and $0.01 \mathrm{M}$ of ACA $(0.065 \mathrm{~g})$ was added to it. The final mixture was then stirred at 1000 rpm at room temperature using magnetic stirrer. After $1 \mathrm{~h}$ of stirring, ACA was completely dissolved and the solution became transparent. Upon $3 \mathrm{~h}$ of additional stirring, the transparent solution started to become brown in color indicating the formation of PbS NPs. After $12 \mathrm{~h}$, these NPs were centrifuged, rinsed many times with distilled water and methanol, and dried in a vacuum oven at $50^{\circ} \mathrm{C}$ for $2 \mathrm{~h}$. Washed and dried PbS NPs were redispersed ultrasonically in methanol for TEM, SAED and Uv-vis characterization.

\subsection{Instruments and Characterizations}

SEM images were recorded on Jeol JSM-6360/LV scanning electron microscope. TEM and SAED were done using a JEOL JEM 1230 electron microscope operated at $120 \mathrm{kV}$. The XRD data was recorded on a Siemens D$5000 \mathrm{X}$-ray diffractometer, using $\mathrm{Cu}-\mathrm{K} \alpha$ radiation $(\lambda=$ $1.54059 \AA)$. The Uv-vis absorption spectrum of agglomerated NPs was recorded on Varian Cary 500 Scan Uvvis spectrophotometer and the specific surface area was calculated from the linear part of the BET equation. The pore diameter distribution was obtained from the analysis of the desorption branch of the isotherms using BarrettJoyner-Halenda (BJH) model. TGA characterisation was carried out from $50^{\circ} \mathrm{C}$ to $650^{\circ} \mathrm{C}$ using a TA, Q-5000 IR instrument with a heating rate of $10^{\circ} \mathrm{C} / \mathrm{min}$. FTIR spectroscopy characterization was obtained using a Nicolet (Thermo Fisher) Model 380 FTIR with an attenuated total reflectance (ATR) sampling device (Smart Performer) with a ZnSe crystal. XPS measurements were carried out on Axis Ultra Kratos X-ray photoelectron spectrometer under a vacuum of $2 \times 10^{-8}-5 \times 10^{-8}$ Torr; the binding energy values were charge-corrected to the $\mathrm{C} 1 \mathrm{~s}$ signal $(285.0 \mathrm{eV})$.

\section{Results and Discussion}

Figure 1 shows electron microscopic images, SAED and XRD patterns of ACA-capped PbS NPs. SEM image (Figure 1(a)) clearly shows the well-defined porous network of NPs agglomeration. TEM image (Figure 1(b)) of methanolic dispersion of ACA-capped PbS NPs shows that these NPs are well dispersed and spherical in shape with an average diameter of around $10 \mathrm{~nm}$. The SAED pattern (inset of Figure 1(b)) shows concentric rings with bright spots, which indicates the nanocrystalline nature of $\mathrm{PbS}$ NPs. All diffraction peaks shown in Figure 1(c) match well with the standard XRD lines of face-centered cubic PbS (JCPDS Card No. 05-0592) and are due to reflections from (111), (200), (220), (311), (222), (400), (331), (420), (422) and (511) planes. Furthermore, no other peaks rather than those corresponding to $\mathrm{PbS}$ are observed,
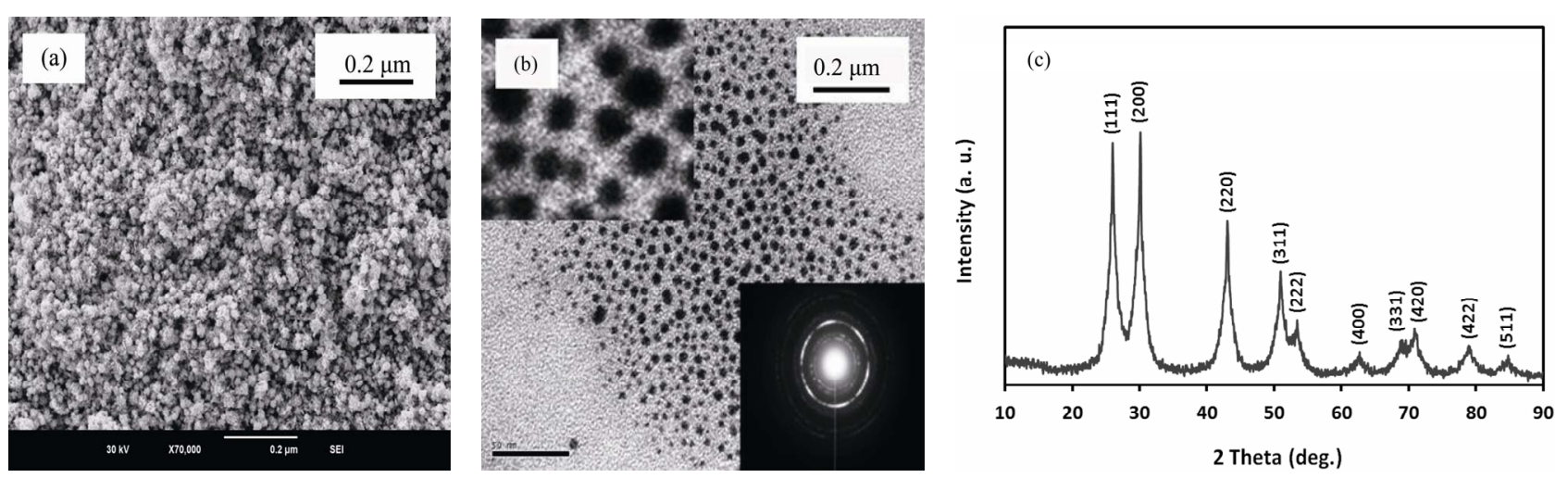

Figure 1. (a) SEM image; (b) TEM image with SAED; and (c) powder XRD pattern of ACA-capped PbS NPs. 
which indicates the high purity of the synthesized $\mathrm{PbS}$ NPs. The broadening peaks indicate that crystals are in the nanoscale size, as confirmed by the TEM observation. Crystal size was estimated from the broadening peak width of $\mathrm{PbS}$ (111) X-ray spectral peaks using the Scherrer formula [23]. It was around $9.2 \mathrm{~nm}$, which is also in close accordance with the size observed by TEM.

The optical absorption spectrum of methanolic ACAcapped PbS NPs is shown in Figure 2. As shown, these NPs absorb strongly in visible region due to their strong size quantization, compared to bulk PbS.

To examine the specific surface area and pore size distribution, $\mathrm{N}_{2}$ adsorption-desorption isotherm measurements were performed on ACA-capped PbS NPs agglomerations in powder form. Figure 3 shows $\mathrm{N}_{2}$ adsorption-desorption isotherm with a hysteretic loop in the range of $0.6 \mathrm{P} / \mathrm{P}_{0}-1.0 \mathrm{P} / \mathrm{P}_{0}$, which indicates that they have a mesoporous structure. Pore size distribution obtained from BJH model is shown in the inset of Figure 3. The corresponding specific surface area is $101 \mathrm{~m}^{2} / \mathrm{g}$.

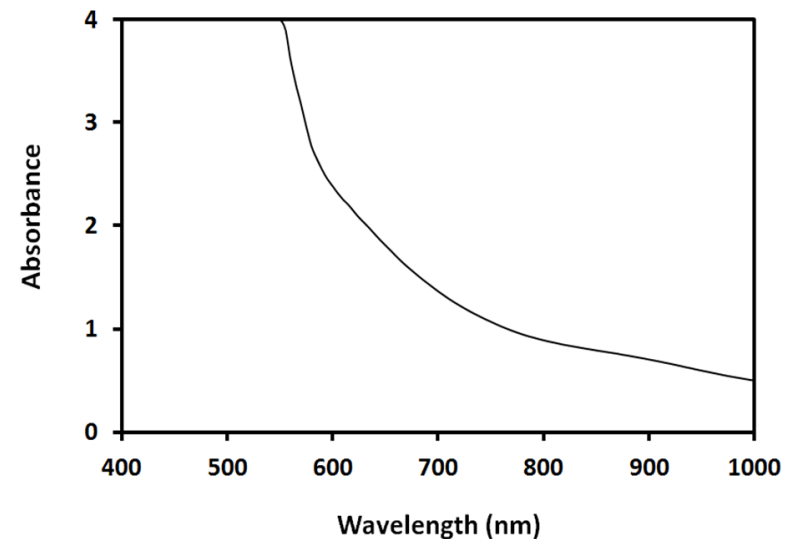

Figure 2. Optical absorption spectrum of ACA-capped PbS NPs.

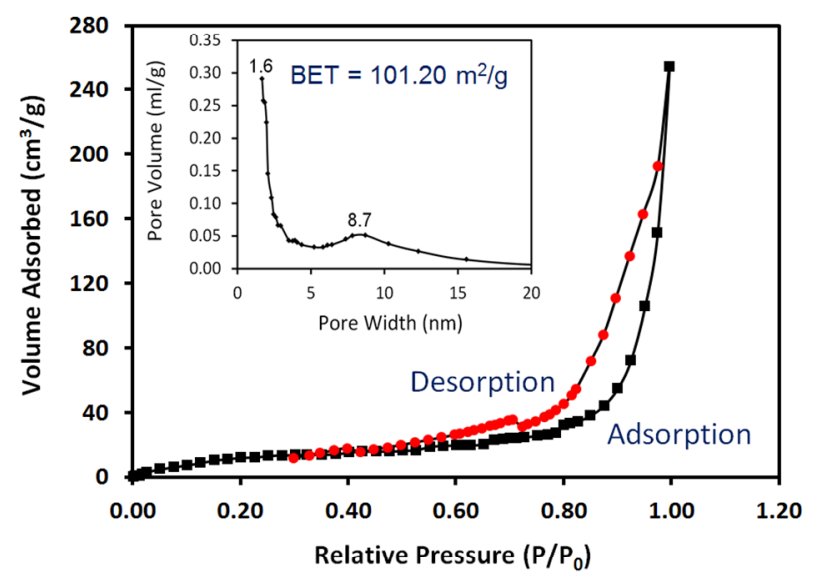

Figure 3. Nitrogen adsorption and desorption isotherms and inset BJH pore radius distribution of ACA-capped PbS NPs powder.
Thermal characterisation from $50^{\circ} \mathrm{C}$ to $650^{\circ} \mathrm{C}$ of synthesized ACA-capped PbS NPs powder is summarized in the TGA plot of Figure 4, obtained at a heating rate of $10^{\circ} \mathrm{C} / \mathrm{min}$ under air atmosphere. The initial weight-loss of around $4 \mathrm{wt} \%$ starting from about $80^{\circ} \mathrm{C}$ corresponds to the water absorbed on the surface of the NPs. The weightloss $(\sim 23 \mathrm{wt} \%)$ observed in the range of $150^{\circ} \mathrm{C}$ to $450^{\circ} \mathrm{C}$ corresponds to the combustion and elimination of ACA molecules. This means that the NPs are composed of about $23 \mathrm{wt} \%$ of ACA and $73 \mathrm{wt} \%$ of PbS NPs. The relatively rapid weight loss in the TGA curve is an indication of a weak binding of ACA molecules to the $\mathrm{Pb}$ atom surface.

FTIR spectra of pure ACA and ACA-capped PbS NPs powder were measured to investigate the interaction between ACA and PbS NPs. The corresponding results are respectively shown by curves (a) and (b) in Figure 5 .

Bands at $2850 \mathrm{~cm}^{-1}-2950 \mathrm{~cm}^{-1}$ are due to C-H stretching vibrations of methylene groups of the ACA carbon chain for both pure ACA and ACA-capped PbS NPs.

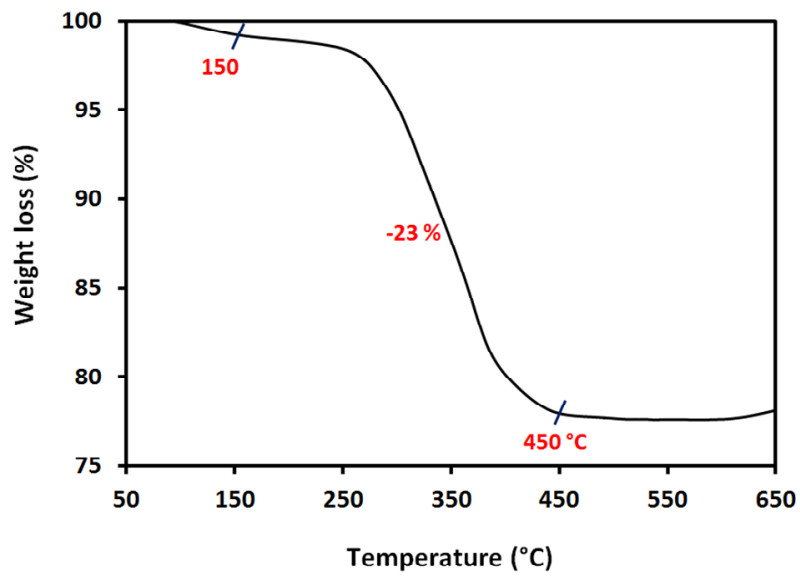

Figure 4. TGA curve of ACA-capped PbS NPs powder.

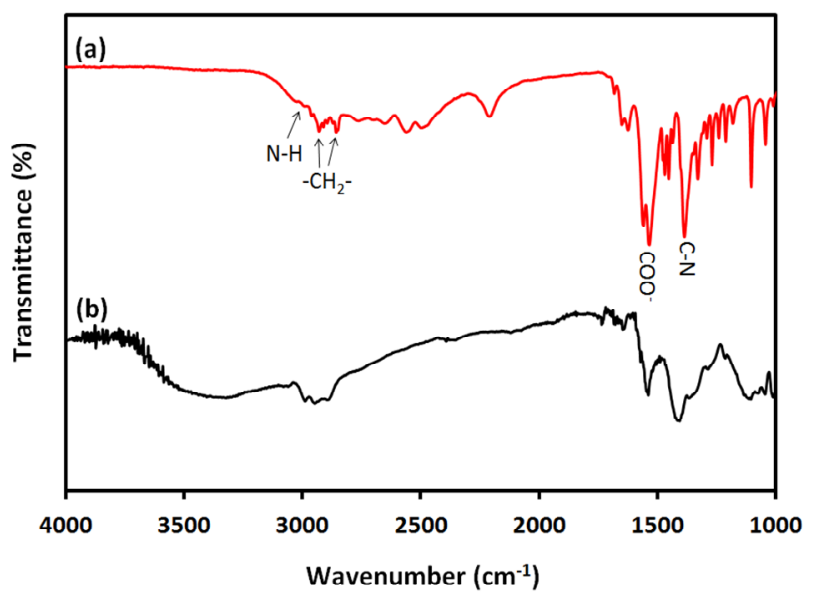

Figure 5. FTIR spectra of (a) ACA and (b) ACA-capped PbS NPs. 
The two bands at $1380 \mathrm{~cm}^{-1}$ and $3050 \mathrm{~cm}^{-1}$ are respecttively due to C-N and N-H stretching modes of ACA molecules. These bands also exist in the FTIR spectrum of ACA-capped PbS NPs at same positions, which indicates the binding of amino $\left(-\mathrm{NH}_{2}\right)$ groups to the PbS NPs surface through nitrogen lone pair. In FTIR spectrum of ACA, the two bands at $1530 \mathrm{~cm}^{-1}$ and $1545 \mathrm{~cm}^{-1}$ are attributed to the symmetric and asymmetric stretching vibrations of uncoordinated -COO- terminal groups of ACA. The spectrum of ACA-capped PbS NPs also shows two bands at the same wavenumbers, which proves that only amino $\left(-\mathrm{NH}_{2}\right)$ groups of ACA molecules were bounded on the $\mathrm{PbS}$ surface and the free carboxylic $(-\mathrm{COOH})$ groups were oriented outward. Furthermore, the fact that no band appears at $1630 \mathrm{~cm}^{-1}$ in the FTIR spectrum of ACA-capped PbS NPs indicates the absence of peptide bond between $\mathrm{C}=\mathrm{O}$ and $\mathrm{C}-\mathrm{N}$ moieties, which was usually observed in literature for amino acid capped NPs produced using amino acids [21]. Also, the broad band around $3300 \mathrm{~cm}^{-1}$ to $3600 \mathrm{~cm}^{-1}$ on curve (b) is due to the presence of adsorbed ater in ACA-capped PbS NPs powder sample. Since no free ACA molecules were left on the surface of the NPs, there is no possibility for peptide formation. It is well known that polypeptide chains are always formed through the interaction between uncoordinated amino acid molecules in solution phase. Therefore, the concentration level of amino acid has an important effect on the final morphology of the synthetized nanomaterials. It is known that high concentration of amino acid in bulk precursor affects the self-assembly of the peptide structure and subsequently affects the morphology of the nanomaterials. From FTIR results, it can be concluded that only amino $\left(-\mathrm{NH}_{2}\right)$ groups of ACA molecules bind on the surface of $\mathrm{PbS}$ NPs via nitrogen lone pair, while carboxylic $(-\mathrm{COOH})$ groups are located at the other side of the ACA chain.

For further examination of the chemical structure of ACS-capped PbS NPs, XPS spectra of Pb 4f, S 2p, C 1s, $\mathrm{O} 1 \mathrm{~s}$, and $\mathrm{N} 1 \mathrm{~s}$ were obtained (Figure 6). The XPS spectrum presented in Figure 6(a) shows that two $\mathrm{Pb}$ peaks appeared at $138 \mathrm{eV}\left(\mathrm{Pb} \mathrm{4f}_{7 / 2}\right)$ and $142.9 \mathrm{eV}\left(\mathrm{Pb} \mathrm{4f}_{5 / 2}\right)$. Figure 6(b) shows that two peaks corresponding to $\mathrm{S}$ $2 p_{3 / 2}$ and $S 2 p_{1 / 2}$ appeared respectively at 161.1 and 162 $\mathrm{eV}$. All binding energy values for $\mathrm{Pb} 4 \mathrm{f}$ and $\mathrm{S} 2 \mathrm{p}$ are slightly higher than what was mentioned in literature for bulk PbS [24]. The difference could be attributed to the coordination action of $\mathrm{Pb}$ moiety and ACA molecules. The $\mathrm{C}$ 1s region (Figure 6(c)) consists of three XPS pea$\mathrm{ks}$ at $284.9,285.8 ., 288.3 \mathrm{eV}$ respectively assigned to C-C, C-N, C-C=O groups of ACA acid molecules [25]. The single and symmetric peak of $\mathrm{O} 1 \mathrm{~s}$ at $531.8 \mathrm{eV}$ (Figure 6(d)) indicates the presence of two symmetric oxygen atoms in of the carboxylic acid moiety. The XPS of $\mathrm{N}$ 1s (Figure 6(e)) shows broad peak around $399.8 \mathrm{eV}$, which is assigned to the nitrogen atom of $-\mathrm{NH}_{2}$ moiety.

It was reported in literature that $\mathrm{PbAc}-\mathrm{TU}$ complex is metastable in methanol and the decomposition of simple methanolic lead acetate-thiourea complex takes place by itself at room temperature leading to $\mathrm{PbS}$ particles [22]. At an initial stage, $-\mathrm{NH}_{2}$ moiety of ACA interacts with $\mathrm{Pb}$ surface through lone pair in methanolic medium. The interaction of $\mathrm{ACA}$ on $\mathrm{Pb}$ surface probably decreases the
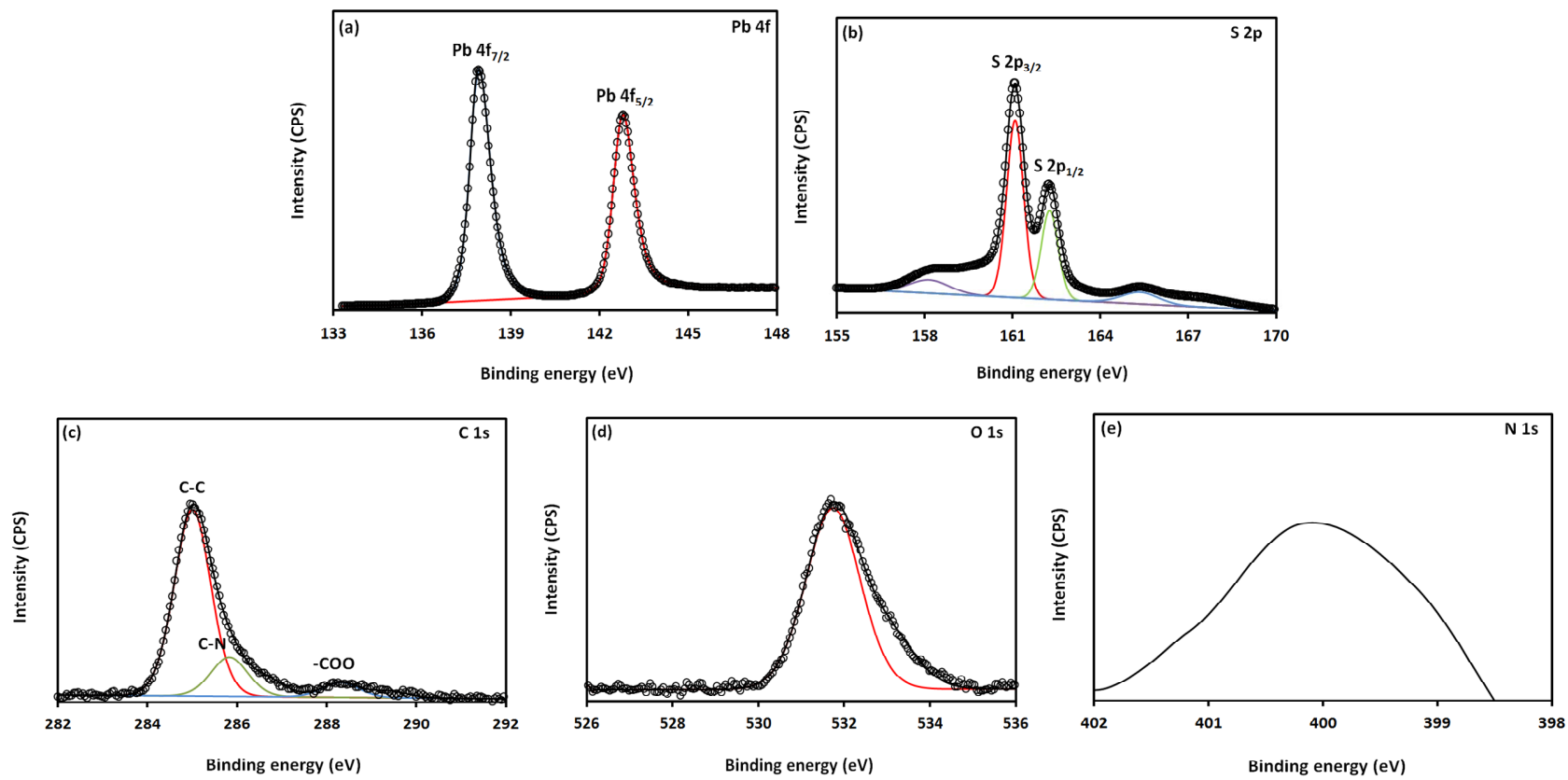

Figure 6. XPS spectra obtained from the ACA-capped PbS NPs: (a) Pb 4f core level; (b) S 2p core level; (c) C 1s core level; (d) $\mathrm{O}$ 1s core level; and (e) $\mathrm{N}$ 1s core level. 
rate of decomposition of PbAc-TU complex. However, this decomposition is further accelerated by continuous stirring. In the reaction mechanism, stirring is a driving force of the reaction. It provides an exposure for the deomposition of PbAc-TU complex. Apart from that, stirring also provides a favorable condition for heterogeneous nucleation, which is ultimately responsible for obtaining regular size and shape of NPs.

\section{Conclusion}

In summary, a simple soft-chemical route for room temperature synthesis of ACA-capped PbS NPs using ACA mixed PbAC-TU complex as a precursor has been developed. SEM, TEM, SAED, Uv-vis and BET studies show that PbS NPs developed via this technique are nanocrystalline, size quantized and present a mesoporous structure. TGA, FTIR and XPS studies of these NPs indicate that ACA acts as a soft template to restrict the growth of PbS NPs through its soft binding via nitrogen lone pair.

\section{Acknowledgements}

The authors would like to thank the Natural Sciences and Engineering Research Council of Canada (NSERC) for financial support of this work.

\section{REFERENCES}

[1] C. B. Murray, D. J. Norris and M. G. Bawendi, "Synthesis and Characterization of Nearly Monodisperse CdE (E = Sulfur, Selenium, Tellurium) Semiconductor Nanocrystallites," Journal of American Chemical Society, Vol. 115, No. 19, 1993, pp. 8706-8715. doi:10.1021/ja00072a025

[2] D. J. Milliron, S. M. Hughes, Y. Cui, L. Manna, J. Li, L. W. Wang and A. P. Alivisatos, "Colloidal Nanocrystal Heterostructures with Linear and Branched Topology," Nature, Vol. 430, No. 6966, 2004, pp. 190-195. doi:10.1038/nature02695

[3] T.-W. F. Chang, S. Musikhin, L. Bakueva, L. Levina, M. A. Hines, P. W. Cyr and E. H. Sargent, "Efficient Excitation Transfer from Polymer to Nanocrystals," Applied Physics Letters, Vol. 84, No. 21, 2004, pp. 4295-4297. doi:10.1063/1.1755414

[4] W. C. W. Chan and N. Shuming, "Quantum Dot Bioconjugates for Ultrasensitive Nonisotopic Detection," Science, Vol. 281, No. 5385, 1998, pp. 2016-2018. doi:10.1126/science.281.5385.2016

[5] Y. Wang, A. Suna, W. Mahler and R. Kasowski, "PbS in Polymers. From Molecules to Bulk Solids," Journal of Chemical Physics, Vol. 87, No. 12, 1987, pp. 7315-7322. doi:10.1063/1.453325

[6] A. A. R. Watt, D. Blake, J. H. Warner, E. H. Thomsen, E. L. Tavenner, H. Rubinsztein-Dunlop and P. Meredith, "Lead Sulfide Nanocrystal: Conducting Polymer Solar Cells," Journal of Physics D: Applied Physics, Vol. 38,
No. 12, 2005, pp. 2006-2012. doi:10.1088/0022-3727/38/12/023

[7] A. Guchhait, A. K. Rath and A. J. Pal, "To Make Polymer: Quantum Dot Hybrid Solar Cells NIR-Active by Increasing Diameter of PbS Nanoparticles," Solar Energy Materials and Solar Cells, Vol. 95, No. 2, 2011, pp. 651-656. doi:10.1016/j.solmat.2010.09.034

[8] S. A. McDonald, G. Konstantatos, S. Zhang, P. W. Cyr, E. J. D. Klem, L. Levina and E. H. Sargent, "SolutionProcessed PbS Quantum Dot Infrared Photo-Detectors and Photo-Voltaics," Nature Materilas, Vol. 4, No. 2, 2005, pp. 138-142. doi:10.1038/nmat1299

[9] P. T. Guerreiro, S. Ten, N. F. Borrelli, J. Butty and G. E. Jabbour, "PbS Quantum-Dot Doped Glasses as Saturable Absorbers for Mode Locking of a Cr:Forsterite Laser," Applied Physics Letters, Vol. 71, No. 12, 1997, pp. 15951597. doi:10.1063/1.119843

[10] Z. H. Zhang, S. H. Lee, J. J. Vittal and W. S. Chin, “A Simple Way to Prepare PbS Nanocrystals with Morphology Tuning at Room Temperature," Journal of Physical Chemistry B, Vol. 110, No. 13, 2006, pp. 6649-6654. doi:10.1021/jp057271m

[11] D. Berhanu, K. Govender, D. Smyth-Boyle, M. Archbold, D. P. Halliday and P. O'Brien, "A Novel Soft Hydrothermal (SHY) Route to Crystalline PbS and CdS Nanoparticles Exhibiting Diverse Morphologies," Chemical Communications, Vol. 45, No. 45, 2006, pp. 4709-4711. doi: $10.1039 / \mathrm{b} 612934 \mathrm{j}$

[12] E. Leontidis, M. Orphanou, T. Kyprianidou-Leodidou, F. Krumeich and C. Walter, "Composite Nanotubes Formed by Self-Assembly of PbS Nanoparticles," Nano Letters, Vol. 3, No. 4, 2003, pp. 569-572. doi:10.1021/n1034124w

[13] K. Singh, A. A. McLachlan and D. Gerrard Marangoni, "Effect of Morphology and Concentration on Capping Ability of Surfactant in Shape Controlled Synthesis of PbS Nano- and Micro-Crystals," Colloids and Surfaces A: Physicochemical and Engineering Aspects, Vol. 345, No. 1-3, 2009, pp. 82-87. doi:10.1016/j.colsurfa.2009.04.033

[14] Z. Zhao, K. Zhang, J. Zhang, K. Yang, C. He, F. Dong and B. Yang, "Synthesis of Size and Shape Controlled PbS Nanocrystals and Their Self-Assembly," Colloids and Surfaces A: Physicochemical and Engineering Aspects, Vol. 355, No. 1-3, 2010, pp. 114-120. doi:10.1016/j.colsurfa.2009.12.009

[15] N. B. Pendyala and K. S. R. Koteswara Rao, "Temperature and Capping Dependence of NIR Emission from PbS Nano-Microcrystallites with Different Morphologies," Materials Chemistry and Physics, Vol. 113, No. 1, 2009, pp. 456-461. doi:10.1016/i.matchemphys.2008.07.125

[16] G. Li, C. Li, H. Tang, K. Cao and J. Chen, "Controlled Self-Assembly of PbS Nanoparticles into Macrostar-Like Hierarchical Structures," Materials Research Bulletin, Vol. 46, No. 7, 2011, pp. 1072-1079. doi:10.1016/j.materresbull.2011.03.006

[17] S. Z. Liu, S. L. Xiong, K. Y. Bao, J. Cao and Y. T. Qian, "Shape-Controlled Preparation of PbS with Various Dendritic Hierarchical Structures with the Assistance of 1-Methionine," Journal of Physical Chemistry C, Vol. 113, 
No. 30, 2009, pp. 13002-13007. doi:10.1021/jp8104437

[18] T. Thongtem, S. Kaowphong and S. Thongtem, "Biomolecule and Surfactant-Assisted Hydrothermal Synthesis of PbS Crystals," Ceramics International, Vol. 34, No. 7, 2008, pp. 1691-1695. doi:10.1016/j.ceramint.2007.05.007

[19] Z. Fan, S. Yan, B. Zhang, Y. Zhao and Y. Xie, "L-Cysteine-Assisted Synthesis of $\mathrm{PbS}$ Nanocube-Based Pagoda-Like Hierarchical Architectures," Journal of Physical Chemistry C, Vol. 112, No. 8, 2008, pp. 2831-2835. doi:10.1021/jp0766149

[20] X. Shen, Z. Li, Y. Cui and Y. Pang, "Glutathione-Assisted Synthesis of Hierarchical PbS via Hydrothermal Degradation and Its Application in the Pesticidal Biosensing," International Journal of Electrochemical Science, Vol. 6, No. 8, 2011, pp. 3525-3535.

[21] T. D. Nguyen, D. Mrabet, T. T. D. Vu, C. T. Dinh and T. O. Do, "Biomolecule-Assisted Route for Shape-Controlled Synthesis of Single-Crystalline $\mathrm{MnWO}_{4}$ Nanopar- ticles and Spontaneous Assembly of Polypeptide-Stabilized Mesocrystal Microspheres," CrystEngComm, Vol. 13, No. 5, 2011, pp. 1450-1460. doi:10.1039/c0ce00091d

[22] T. Chaudhuri, N. Saha and P. Saha, "Deposition of PbS Particles from a Nonaqueous Chemical Bath at Room Temperature," Materials Letters, Vol. 59, No. 17, 2005, pp. 2191-2193. doi:10.1016/j.matlet.2005.02.064

[23] Y. Zhao, X.-H. Liao, J.-M. Hong and J.-J. Zhu, "Synthesis of Lead Sulfide Nanocrystals via Microwave and Sonochemical Methods," Materials Chemistry and Physics, Vol. 87, No. 1, 2004, pp. 149-153. doi:10.1016/j.matchemphys.2004.05.026

[24] G. E. Muilenberg, C. D. Wager, W. M. Riggs, L. E. Devis and J. F. Moulder, "Handbook of X-Ray Photoelectron Spectroscopy," Perking-Elmer, New York, 1979.

[25] Z. Deng, B. Peng, D. Chen, F. Tang and A. Muscat, "A New Route to Self-Assembled Tin Dioxide Nanospheres: Fabrication and Characterization," Langmuir, Vol. 24, No. 19, 2008, pp. 11089-11095. doi:10.1021/la800984g 